\title{
Trouble at depth
}

\author{
The deep ocean is largely uncharted territory. The aftermath of the BP oil spill has been a poignant \\ reminder that its relative inaccessibility hinders exploration, but does not extend much protection from \\ human interference.
}

Interest in marine ecosystems has surged since the BP blowout in the depths of the Gulf of Mexico. Images of oil-drenched pelicans and tar-stained beaches have circulated the globe, and garnered public concern for the health and resilience of marine life. Research scientists descended on the Gulf in the wake of the disaster to monitor the fate of the spill - not just at the surface, where the effects are most apparent - but also deep within the ocean interior and at the sea floor. But getting even a rough idea what is going on beneath the surface of the ocean is a formidable task.

Monitoring the fallout from the spill is proving tricky. Observations that have been published contradict each other. One study reports the presence of a $35-\mathrm{km}$ long subsurface plume showing few signs of decay (Science Express doi:10.1126/ science.1195223; 2010). Another provides evidence for a vigorous community of hydrocarbon-degrading microbes that are eating their way through the oil (Science Express doi:10.1126/science.1195979; 2010).

However, both studies are relatively small in scale and may describe just local conditions. What seems to be missing is a comprehensive overview of all existing observations, and a coordinated effort to fill any important gaps in the coverage (Nature $467,16 ; 2010)$. But since the oil plume vanished from the surface, and hence the view of satellite sensors, locating the spill has turned into a challenge: research vessels can make only point measurements in a restricted area, and launching instruments to great depth takes time.

Oil spill researchers are not alone in their difficulties in charting the deep ocean. The Census of Marine life - a ten-year project set up to study the diversity, distribution and abundance of life in the seas - has made only the smallest contribution to our knowledge of marine species. Despite a decade of intensive effort, the sum total of census activities led to the description of about 1,200 new species - and left an estimated 700,000 marine animals and plants to be classified by posterity (Commentary, page 662). Of course, reports of outlandish

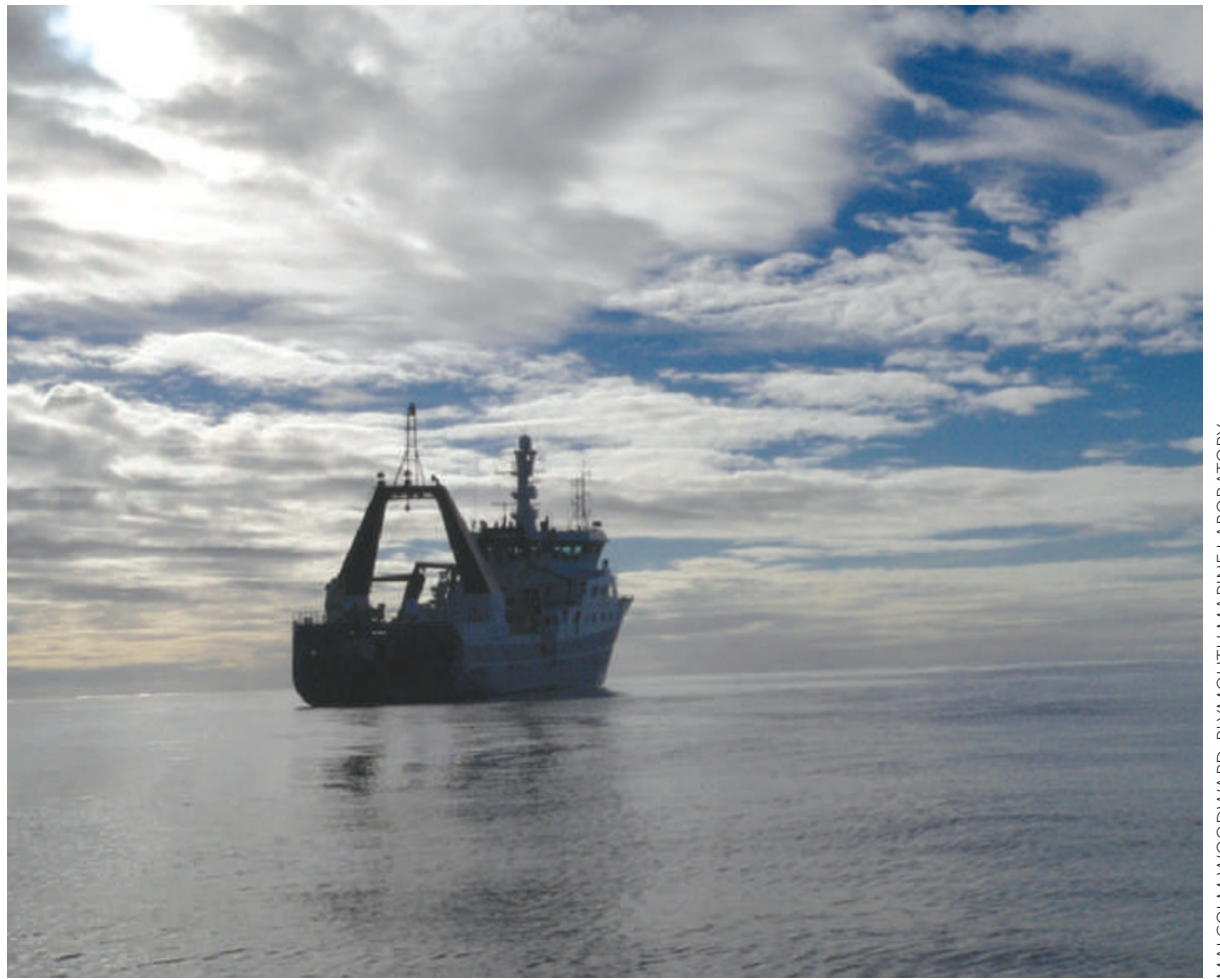

newly discovered organisms and news of biodiversity hotspots have elicited much media interest. But many habitats, including vast swathes of the deep ocean, have barely been sampled.

Perhaps the Census of Marine Life could have progressed somewhat more quickly with a different approach to the problem. The development of automated methods for identifying new species may have helped. Taxonomy, the branch of science responsible for the identification, description and naming of new species, is an underfunded, highly specialized art, where the low number of experts capable of diagnosing new species limits progress. Automated identification systems could potentially yield more consistent identifications in less time (Nature 467, 154-155; 2010). And the organizational structure of the census may not have been ideal for the task at hand (Commentary, page 662). But fundamentally, the inaccessibility of the deep ocean - a dark, cold, high-pressure and extremely corrosive environment that can be reached only by ship at great expense - has slowed progress.

Yet the Census of Marine Life has provided an observation that turns out to be poignant in the light of recent events. The pre-blowout Gulf of Mexico was listed as one of the most biodiverse oceanic regions on our globe (PLoS ONE doi:10.1371/journal.pone.0012110; 2010). Whether this will remain true once the toxic hydrocarbons, oxygen-depleting gases and chemical dispersants have taken their toll is one of the questions that need to be answered in the aftermath of the disaster.

Elucidating the long-term impacts of the spill will not be easy. But if technology allowed us to drill a borehole in the sea floor at a depth of over $10,000 \mathrm{~m}$, it is hard to imagine that it would be impossible to find out what damage the accident has done, given adequate resources. 